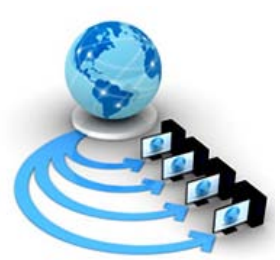

Volume 9, No. 2, March-April 2018

International Journal of Advanced Research in Computer Science

RESEARCH PAPER

\author{
Available Online at www.ijarcs.info
}

\title{
COMPARATIVE STUDY OF DIFFERENT ACTION FPS AND PUZZLE GAMES
}

\author{
Priya Bharambe \\ BE Student \\ Department of Information Technology \\ Vidyalankar Institute of Technology, \\ Mumbai, India \\ Aditi Patil \\ BE Student \\ Department of Information Technology \\ Vidyalankar Institute of Technology, \\ Mumbai, India
}

\author{
Sumit Kumar Sharma \\ BE Student \\ Department of Information Technology \\ Vidyalankar Institute of Technology, \\ Mumbai, India \\ Ichhanshu Jaiswal \\ Assistant Professor \\ Department of Information Technology \\ Vidyalankar Institute of Technology, \\ Mumbai, India
}

\begin{abstract}
Video games are an integral part of our lifestyle. Today, various types of video games are played in different parts of the world. They are a medium of communication, entertainment among people. It can make a strong impact on the society, good or bad, however it maybe. This paper emphasizes on the difference between various such games, their play styles, features, advantages and disadvantages, especially on the puzzle and action category games. The focus is on the game categories, their environment and impact on the society. Based on this, a new game can be proposed to attract more players. It will also result in a positive impact by increasing aptitude skills, visual skills as well as reflex action, decision skills.
\end{abstract}

Keywords: FPS, games, video, puzzle, action

\section{INTRODUCTION}

As computer and video games have exponentially increased popularity over time, they have a significant influence on popular culture [1]. Game culture has also evolved over time hand in hand with internet culture as well as the increasing popularity of mobile games. In the current world, where stress has become an important part of life, people of all ages seem to take gaming as a stress buster [2]. A game can have positive as well as negative effect on a human being. It plays an important role in the current lifestyle. The motive behind this paper is to study different games, their features, effects on the society and develop a new game which can outcome the cons along with providing the pros. Hence, we searched, studied different games, and compared them for various features and proposed a new game as a solution.

\section{SURVEY WORK}

There are not many games that are based on more than a single category. The market has more than 10 categories of games, each having their special features, advantages and disadvantages [3].

\section{Counter Strike- Global Offense and Counter Strike:}

These are famous online video games, which are of franchise Counter Strike, are an objective-based, multiplayer first-person shooter games [4]. Two opposing teams, known as the Terrorists and the Counter Terrorists, compete in game modes to complete objectives, such as securing a location to plant or defuse a bomb and rescuing or guarding hostages.

\section{Features:}

The game focuses on MMORPGs (Massively Multiplayer Online Role Playing Games)

It provides large virtual worlds.

It helps in increasing the social connectivity as it connects various players around the world.

\section{Drawbacks}

It is a violent aggressive game.

It results in building of stress.

It develops addiction from the young age causing a severe effect on the lifestyle.

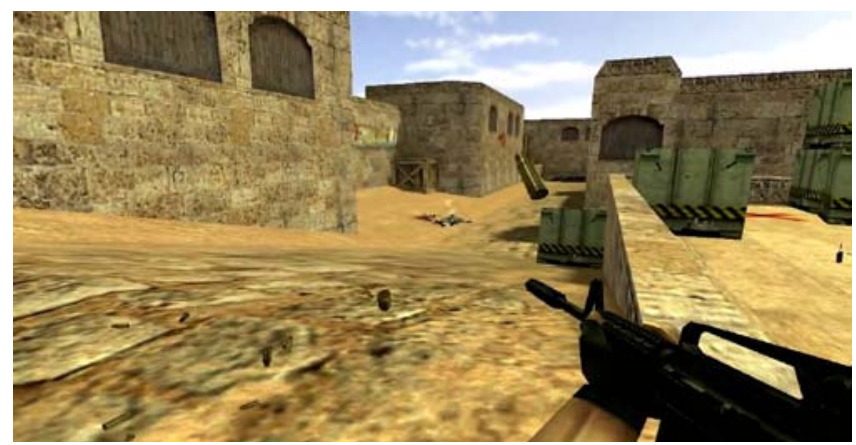

Fig. 1.0 Counter Strike (Game Scene) 


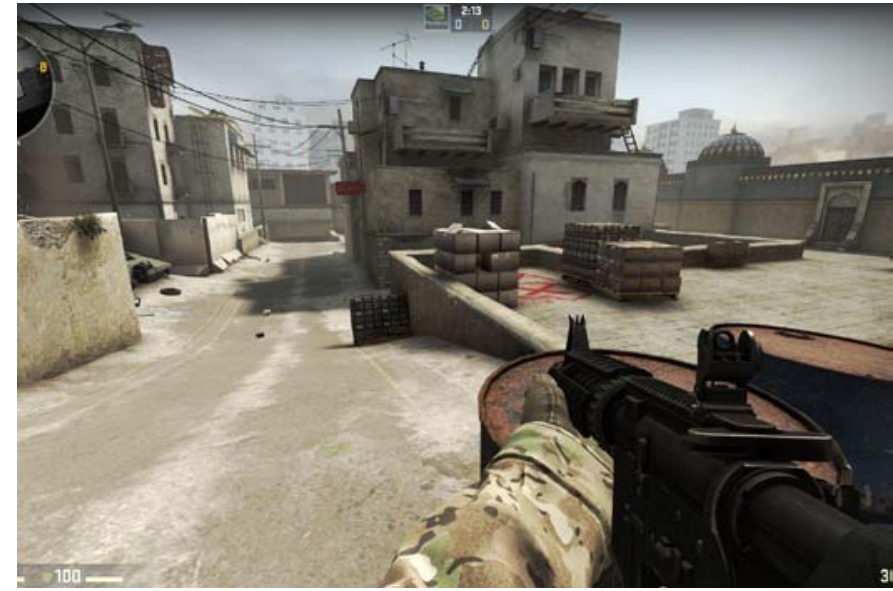

Fig. 1.1 Counter Strike- Global Offensive (Game Scene)

2. Doom:

It is a first peron shooting game in which a player has to play in order to survive from the attacks of the forces of hell i.e. of hordes of demons and undeads [5]. This game is under First Person Shooting genre, which involves same thinking ability of "how to survive".

\section{Features s:}

It provides action, adventure to the player.

It helps in gaining the decision making skills, has good graphics.

Drawbacks:

It supports some mythological controversies of concepts like hell, demons or angels which can make an impact on a child's thinking.

It doesn't help in a good kind of way and can affect mental health of children as it contains much violence and few disturbing components like devils as shown in the figure 2.0 below.

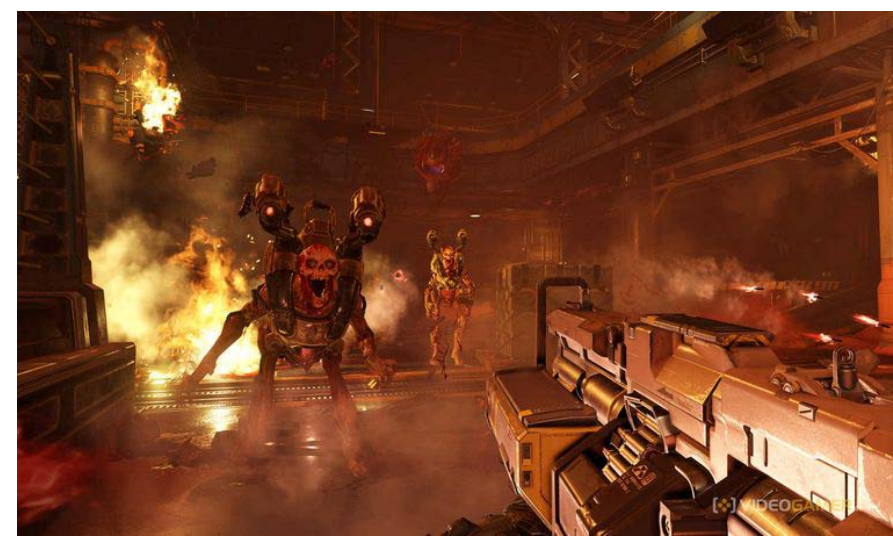

Fig. 2.0. Doom (Game Scene)

\section{FAR CRY}

It is a franchise of first-person shooting video games, all of which have been published by Ubisoft [6]. Until today, there are 5 versions of Far Cry released. Each version follows the concept of "How to Survive". The player is stuck in the given map of wildlife and he needs to survive to win the game. The game is basically single player, but multiplayer options are also available.

\section{Features:}

Far cry has very compelling characters and stories.

It has very good graphics.

There are consistent surprises in the game means it's impossible to predict which makes it interesting.

\section{Drawbacks:}

The wildlife can be unnecessarily aggressive which can result in a strong negative impact.

In order to survive, player has to kill different animals which can result in a bad influence.

The recent releases have a similar structure for the map, as shown in the example below.

In the recent reviews, people have considered this game as a dumb game because of the stories [7].

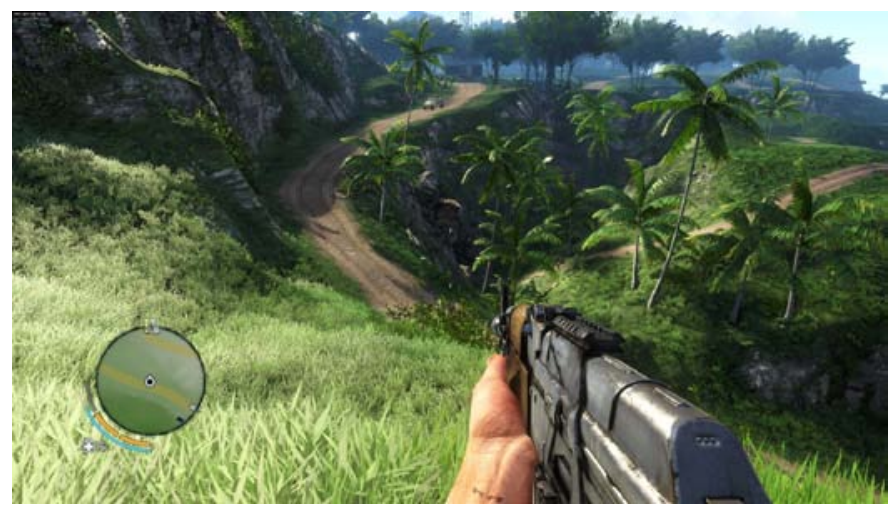

Fig. 3.0 Far Cry 3

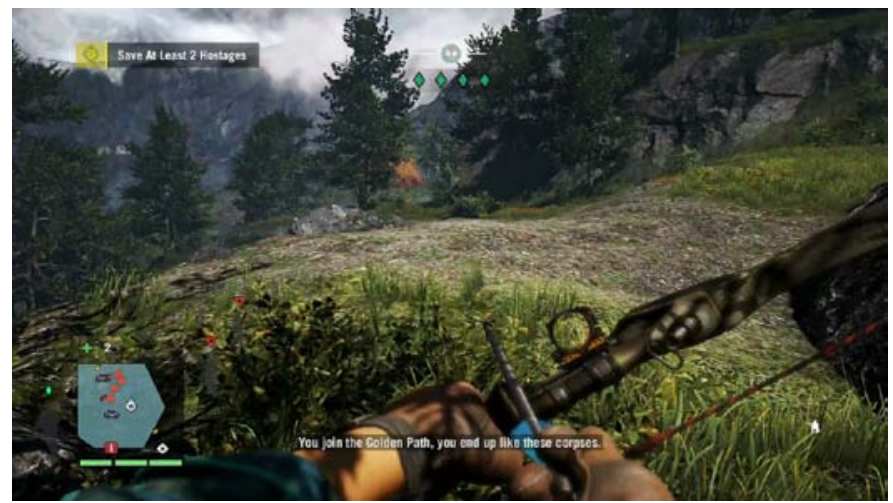

Fig. 3.1 Far Cry 4

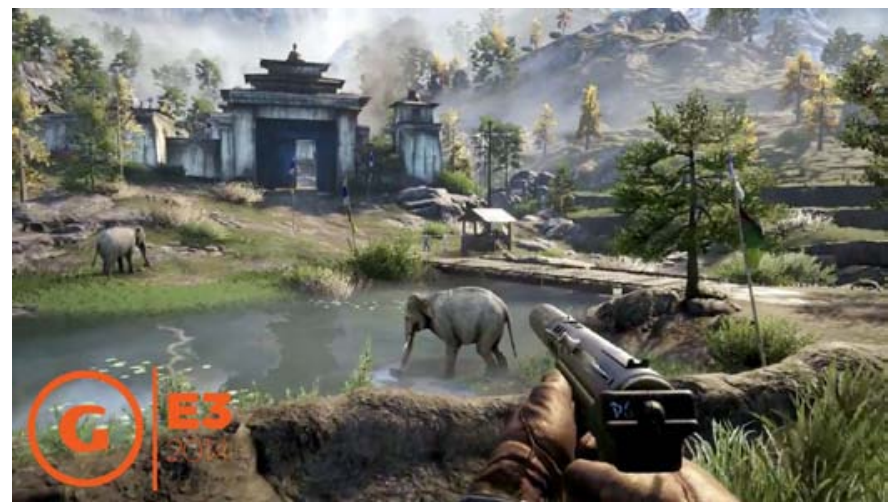

Fig 3.2 Far Cry 4 (Killing an animal)

\section{CRIMINAL CASE}

It is a game where you play as a detective and must find and solve clues to win the game. The game comes under puzzle 
category [8]. It focuses only on one category, i.e. puzzles. The playing environment is a closed room and the player has to find clues in order to go to next level.

\section{Features:}

It helps a person for developing good observing skills.

It can be helpful for developing grasping skills and thinking ability.

\section{Drawbacks:}

There are repeated cases.

It keeps revolving around the room's environment shown in the figure 4.0, which makes it boring after a period of time.

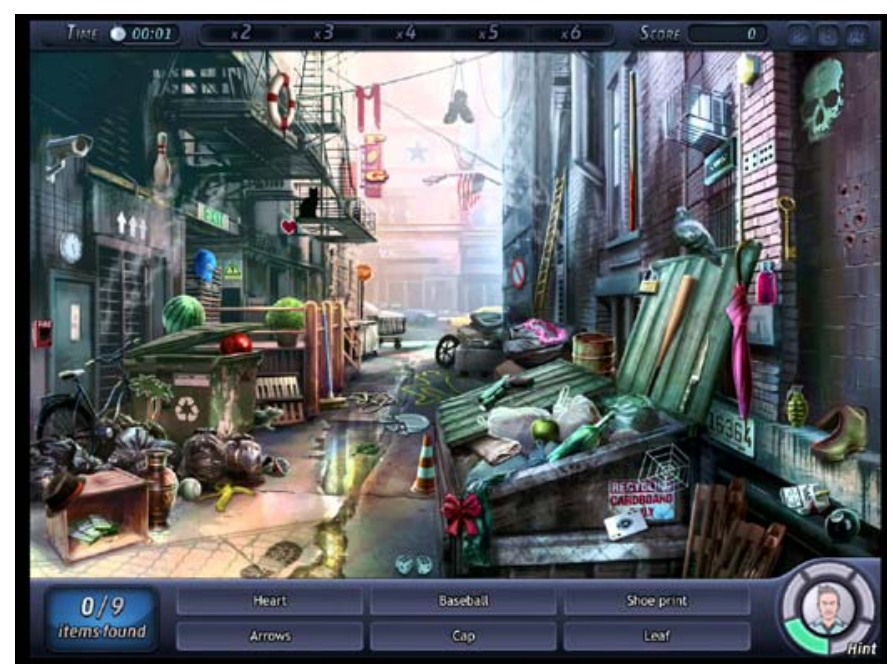

Fig. 4.0 Criminal Case (Level 13 Gameplay)

\section{BEJEWELED}

It is a puzzle category game in which you have to swipe tiles and match them in order to win [9].

\section{Features:}

It provides different levels with little amount of improvised graphics with their new 3 releases.

\section{Drawbacks:}

In the whole game, it follows the only concept of swapping the tiles in order to achieve the target.

For e.g., in the two figures shown below, figure 5.0 shows the basic level and figure 5.1 shows new release with advanced level.

The only difference between them is change of visual scorecard and the form of jewels (normal to butterflies). Hence it becomes boring after a certain stage.

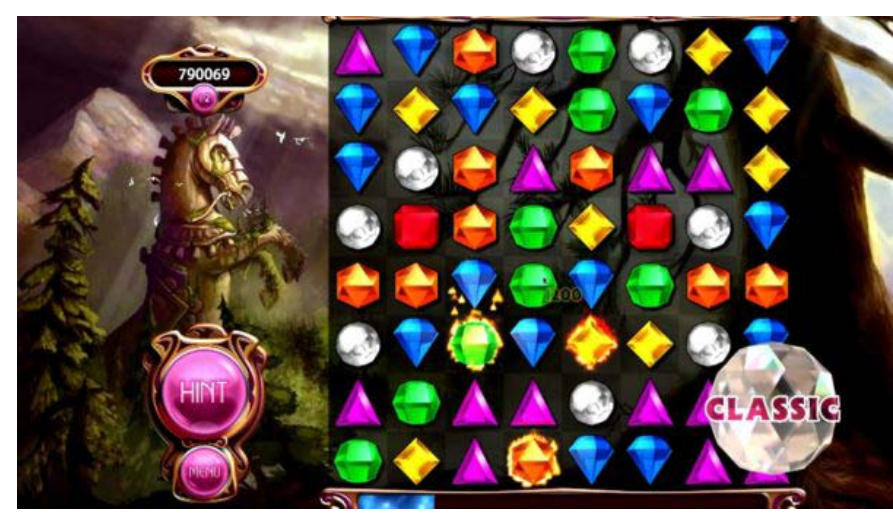

Fig. 4.0 Bejeweled (Basic Level)

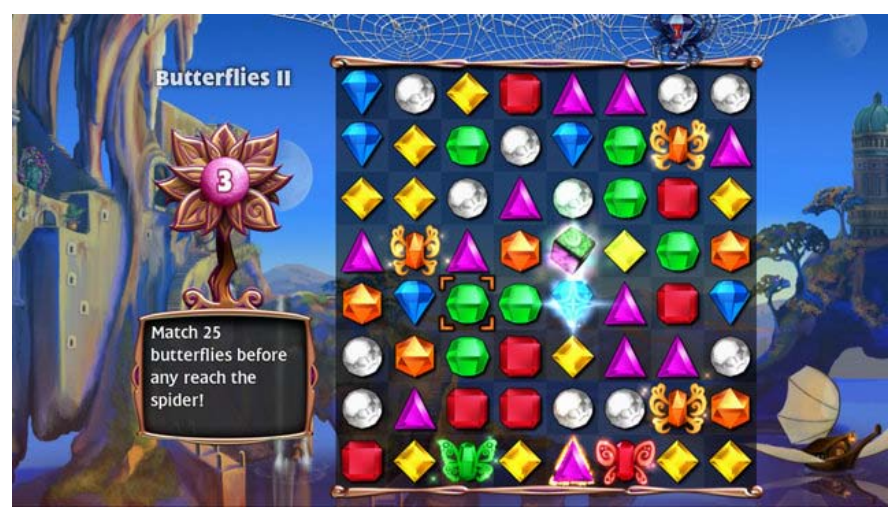

Fig. 4.1 Bejeweled 3 (advanced level)

\section{SOKOBOND}

It is also a puzzle category game which is based on chemistry and movements of blocks [10]. A player has to solve chemistry equations which can be considered as puzzles.

Features:

This provides an interesting sight in chemistry as the player can learn and develop interest in chemistry while playing the game.

The different UI for the equations makes the tedious studies enjoyable.

Drawbacks:

It provides questions related to only chemistry equations i.e. only one subject. Hence, it gets boring after a while. The interface is difficult to understand for new players; hence it gets tedious at the start.

In the end, for more difficult chemistry equation like for breaking a molecular bond, player has to break the tiles which is very difficult and hence after a certain period, one tends to stop playing this games. It can be a frustrating game as per reviewers [11].

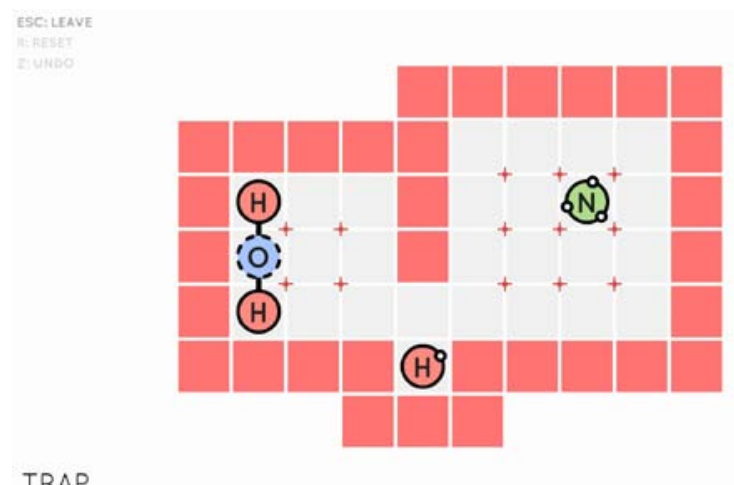

Fig. 6.0 Sokobond Gameplay

\section{THE WITNESS}

The Witness is also a puzzle genre game which is similar to Criminal Case as mentioned before. In this game a player is presented in an island map, in which he/she has to find clues to regain his/her memory, find the way back home [12]. 
Features:

It provides good graphics and user experience.

It improvises player's thinking ability, decision making skills etc.

\section{Drawbacks:}

The levels are limited and basic. For e.g. sand level, water level, mountain level etc.

The game tells the story again and again which makes it more boring and tedious according to the reviews [13].

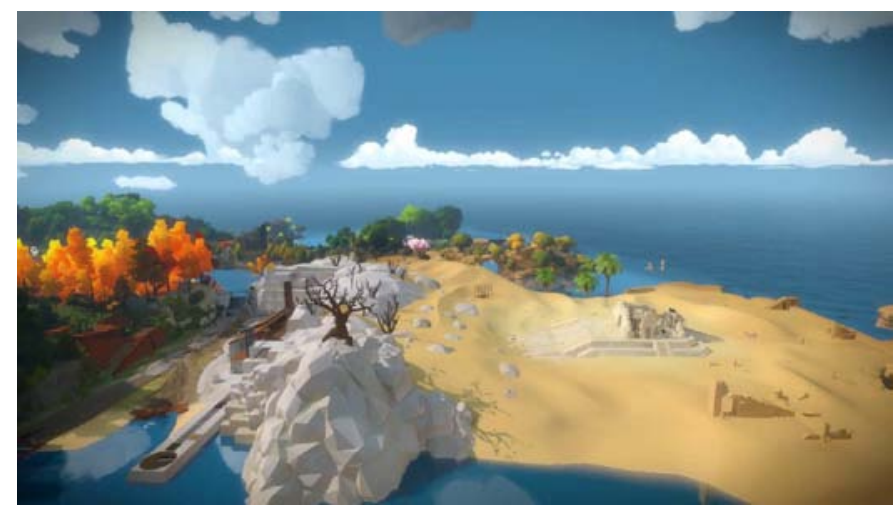

\section{OBSERVATION}

Fig. 7.0 The Witness

Different types of games are available easily on the internet nowadays, through different platforms like steam, bluestacks, GOG etc. Games based on a single category or of a single genre tend to be boring or repeating after a certain time period. Players need some change in a game to make it addictive and interesting. All first person shooting games provide good graphics, maps but are aggressive in nature resulting in developing emotions like anger. They contain violent activities, controversial components which are not good for a young age group of people. The puzzle genre or similar games become addictive but tend to get boring after a certain level since every level follows almost same structure and limited puzzles or questions.

The games which we surveyed have positive as well as negative effects. People tend to use puzzle or question games to increase their knowledge or to make their study interesting, and first person shooting games for role playing experience, adventure and entertainment. But each category of games has pros and cons at last.

\section{PROPOSED SYSTEM}

To make a game more interesting, attractive and playable than the surveyed games, we proposed a game that will come under Puzzle, Action, First Person Shooting genres. Our proposed game will consist of questions i.e. puzzles and a battle ground for final combat, which will combine these three genres to remove cons of being a single cateogry and can serve educational as well as entertainment purpose. Puzzles or questions will be used for gathering knowledge, information and increasing your aptitude skills. The battle ground i.e. a creative map suitable and interesting for every age group will be created for the action part. It will include questions as well as adventure and action.

\section{CONCLUSION}

Although there are many games available in the market, each game specifies a single category and have certain pros and cons as discussed. To decrease the negative impacts, a new game can be proposed using two or more genres to make it more educational and interesting for all the age groups of the society. Hence, we propose a game which focuses on merging two types - puzzles and first person shooter or action into one and creating a product which is entertaining as well as an educational benefit. The proposed game will help one to increase the speed of solving any problem in real time, as the winning and losing depends on the time taken to solve the problem. So a question or puzzle, which results in good progress in something you love, will motivate you to solve the question quickly and effectively, thereby increasing your problem solving and thinking speed. A battle ground, where a unique map will be provided with variety for each level, will make it more interesting along with the questions. Combat at the end in the provided suitable map will give the player an experience of adventure and action which will help in developing decision making abilities, self awareness etc. In overall, the game will provide entertainment along with education which will surely make a positive impact on the society.

\section{REFERENCES}

[1] http://www.streetdirectory.com/travel_guide/103986/gamin g/effects_of_online_gaming_on_kids.html

[2] http://learningworksforkids.com/2017/07/video-gamesprocessing-speed-research-recommendation(Dated 08/09/2017)

[3] https://houghtcatalog.com/jane-hurst/2015/02/12-typesof-computer-games-every-gamer-should-know-about/

[4] www.counter-strike.net/

[5] https://doom.com/en-us/

[6] https://www.ubisoft.com/en-us/game/far-cry-3

[7] https://www.techspot.com/products/pc-games/far-cry4.110475

[8] /https://www.criminalcase.com/

[9] http://zone.msn.com/en/bejeweled/

[10] http://www.sokobond.com/

[11] http://www.dealspwn.com/sokobond-review-steammolecules-177976

[12] http://the-witness.net/

[13] http://mostly-retro.com/2016/02/03/the-witness-review-awitness-to-disaster/ 